\title{
Solar origins of a strong stealth CME detected by Solar Orbiter
}

\author{
Jennifer O'Kane ${ }^{1}$, Lucie M. Green ${ }^{1}$, Emma E. Davies ${ }^{2}$, Christian Möstl ${ }^{3}$, Jürgen Hinterreiter ${ }^{3,4}$, \\ Johan L. Freiherr von Forstner ${ }^{5}$, Andreas J. Weiss ${ }^{3,4}$, David M. Long ${ }^{1}$, and Tanja Amerstorfer ${ }^{3}$ \\ 1 Mullard Space Science Laboratory, UCL, Holmbury St Mary, Dorking, Surrey RH5 6NT, UK \\ e-mail: ucasjro@ucl.ac.uk \\ 2 Department of Physics, Imperial College London, London, UK \\ 3 Space Research Institute, Austrian Academy of Sciences, Graz, Austria \\ 4 Institute of Physics, University of Graz, Graz, Austria \\ 5 Institut für Experimentelle und Angewandte Physik, Christian-Albrechts-Universität zu Kiel, Kiel, Germany
}

Received 21 February 2021 / Accepted 30 March 2021

\begin{abstract}
Aims. We aim to locate the origin of a stealth coronal mass ejection (CME) detected in situ by the MAG instrument on board Solar Orbiter and make connections between the CME observed at the Sun and the interplanetary CME (ICME) measured in situ.

Methods. Remote sensing data were analysed using advanced image processing techniques to identify the source region of the stealth $\mathrm{CME}$, and the global magnetic field at the time of the eruption was examined using potential field source surface models. The observations of the stealth CME at the Sun were compared with the magnetic field measured by the Solar Orbiter spacecraft, and plasma properties were measured by the Wind spacecraft.

Results. The source of the CME is found to be a quiet Sun cavity in the northern hemisphere. We find that the stealth CME has a strong magnetic field in situ, despite originating from a quiet Sun region with an extremely weak magnetic field.

Conclusions. The interaction of the ICME with its surrounding environment is the likely cause of a higher magnetic field strength measured in situ. Stealth CMEs require multi-wavelength and multi-viewpoint observations in order to confidently locate the source region; however, their elusive signatures still pose many problems for space weather forecasting. The findings have implications for Solar Orbiter observing sequences with instruments such as EUI that are designed to capture stealth CMEs.
\end{abstract}

Key words. Sun: coronal mass ejections (CMEs) - Sun: corona - Sun: magnetic fields - Sun: heliosphere

\section{Introduction}

Coronal mass ejections (CMEs) are large eruptions of the Sun's plasma and magnetic field into the heliosphere. If they are Earthdirected, these events have the potential to drive space weather effects. A subset of interplanetary CMEs (ICMEs), known as magnetic clouds, exhibit enhanced magnetic field strength, a rotation in at least one of the magnetic field components, and low plasma density, temperature, and plasma $\beta$ when detected in situ (Burlaga et al. 1981). The helical field configuration of a magnetic cloud is known as a flux rope. The geoeffectiveness of a magnetic cloud (or indeed any CME) is highly dependent on the strength and orientation of the magnetic field. In particular, a strong southward $B_{z}$ for a period of time longer than a few hours typically produces a strong geomagnetic storm. In order to improve space weather prediction capabilities, it is then necessary to study the CME source region and use this knowledge to forecast the configuration and properties of the magnetic field in the interplanetary medium. The source region may or may not have a flux rope configuration itself (see Green et al. 2018; Patsourakos et al. 2020, for reviews). However, if a flux rope is present, it can be identified using a range of remote sensing data and the knowledge used to forecast its geoeffectiveness (see Palmerio et al. 2017, 2018, and references therein).

Stealth CMEs lack the classic low coronal signatures typically associated with CMEs, such as solar flares, extreme ultraviolet (EUV) dimmings, and filament eruptions (see overview by Howard \& Harrison 2013). However, it has been shown that image processing techniques can be used to identify observational signatures, although these can be very faint and hard to detect (Alzate \& Morgan 2017; O'Kane et al. 2019). Studies carried out so far indicate that stealth CMEs may originate at relatively high heights $\approx 1.3 R_{\odot}$ in the corona (Robbrecht et al. 2009; O'Kane et al. 2021), implying that the eruptive structure has a relatively low magnetic field strength and low plasma density, leading to a correspondingly low energy release, lack of strong radiative emissions, and lower propagation speed $\left(<500 \mathrm{~km} \mathrm{~s}^{-1}\right.$, D'Huys et al. 2014). Despite these characteristics, stealth CMEs still have the potential to be geoeffective and therefore present a challenge for space weather forecasting (Kilpua et al. 2014; Nitta \& Mulligan 2017). Carrying out in situ and remote sensing connection studies can help one to understand the origins, evolution, and impact of CMEs in the heliosphere.

Here, we study the solar origins of the first CME detected in situ by Solar Orbiter (Müller et al. 2020). The encounter took place on 19 April 2020 at a heliocentric distance of $0.809 \mathrm{AU}$. The details of the CME's structure and propagation in the inner heliosphere are reported in Davies et al. (2021) and information on the corresponding Forbush decrease can be found in Freiherr von Forstner et al. (2021). The interplanetary CME had a clear flux rope structure and its arrival at Earth produced a Dst 
minimum of $-60 \mathrm{nT}$ on 20 April 2020 and a category G1 geomagnetic storm. At the Sun, the event was determined to be a streamer blowout with no clear signatures in the lower corona. This places the CME in the stealth category and, here, we present the identification and analysis of the CME source region that was made possible using advanced image processing techniques. The paper is organised as follows: Sect. 2 describes the in situ and remote sensing data as well as the methods used within this study; Sect. 3 describes the analysis of the stealth CME connecting the in situ and the solar source perspectives; and finally Sect. 4 discusses the results and conclusions.

\section{Data and methods}

\subsection{Data}

For the in situ analysis, magnetic field data were obtained from the magnetometer (MAG; Horbury et al. 2020) on board Solar Orbiter (Müller et al. 2020), which provides in situ observations of the CME at approximately $0.8 \mathrm{AU}$. The magnetic field investigation (MFI; Lepping et al. 1995) and solar wind experiment (SWE; Ogilvie et al. 1995) of the Wind spacecraft provide magnetic field and solar wind plasma observations at $1 \mathrm{AU}$.

For the remote sensing observations, the white light CME was analysed using data from the Large Angle and Spectrometric COronagraph (LASCO; Brueckner et al. 1995) on board the SOlar and Heliospheric Observatory (SOHO; Domingo et al. 1995) and the COR 1 and 2 coronagraphs part of the Sun Earth Connection Coronal and Heliospheric Investigation instrument suite (SECCHI, Howard et al. 2008) on board the Solar TErrestrial Relations Observatory Ahead (STEREO-A, Kaiser et al. 2008) spacecraft. EUV data were taken from the Atmospheric Imaging Assembly (AIA, Lemen et al. 2012) on board the Solar Dynamics Observatory (SDO, Pesnell et al. 2012) spacecraft, and the Extreme Ultraviolet Imager (EUVI; Howard et al. 2008) on board STEREO-A. Line of sight magnetic field data were taken from the Helioseismic and Magnetic Imager (HMI) data (Scherrer et al. 2012) on board SDO.

\subsection{Methods}

Coronagraph data were processed using the Normalising-RadialGraded Filter (NRGF, Morgan et al. 2006). EUV data were processed using both the Multi-scale Gaussian Normalisation (MGN) technique (Morgan \& Druckmüller 2014) and the NRGF technique. Stack plots were created using COR1 and COR2 data by taking a radial slice from Sun centre. The slice taken at $90^{\circ}$ is presented in this paper, as this most clearly shows the CME curve. The plane-of-sky kinematics were obtained using the Savitzky-Golay bootstrapping technique (Byrne et al. 2013), and the 3D shape and orientation of the CME in the COR2 and LASCO/C2 fields of view were modelled using the graduated cylindrical shell (GCS) model (Thernisien et al. 2006; Freiherr von Forstner 2021). Longitude and latitude are defined in Carrington coordinates.

\section{The stealth CME}

\subsection{In situ observations}

In situ magnetic field signatures observed by both Solar Orbiter and Wind as well as the solar wind plasma data observed by Wind whilst the spacecraft were close to radial alignment, but separated in longitude by less than $5^{\circ}$, are shown in Fig. 1.
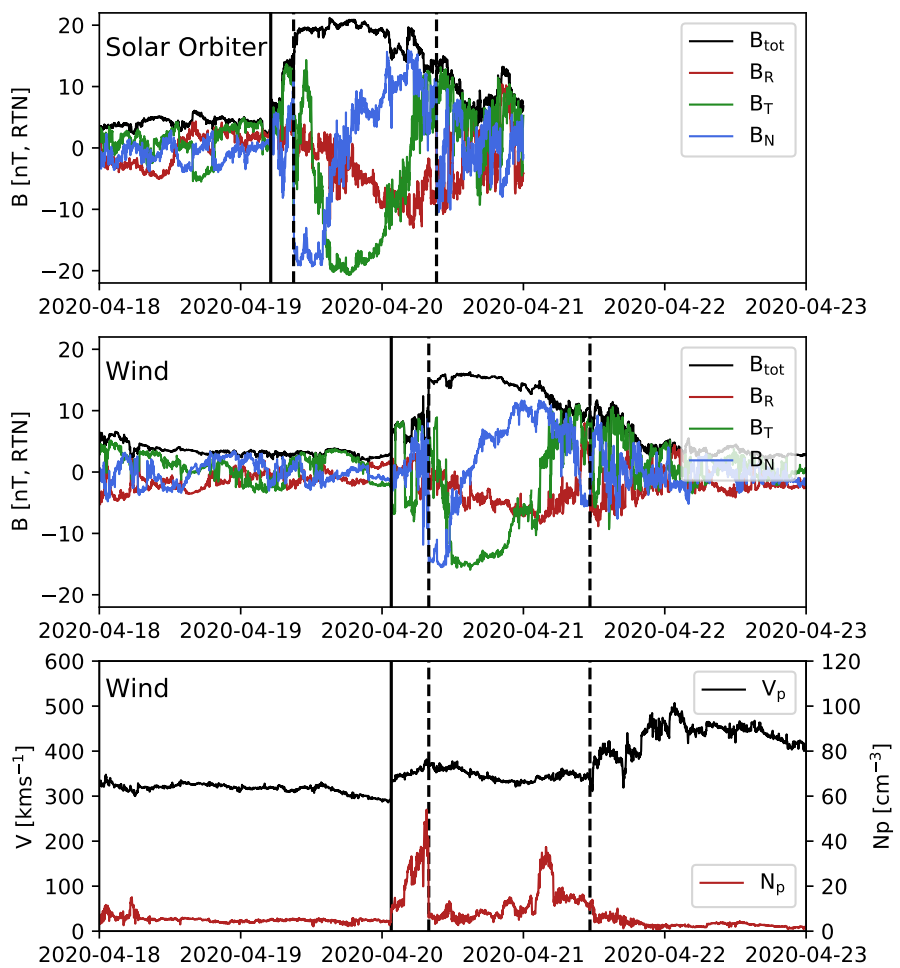

Fig. 1. In situ observations of the CME. In each panel, the shock front is delineated by the solid vertical line, and the magnetic flux rope boundaries are shown by the vertical dashed lines. Top panel: Solar Orbiter magnetic field data in radial-tangential-normal (RTN) coordinates, where the total magnetic field magnitude is shown in black and the magnetic field components, RTN, are in red, green, and blue, respectively. Middle panel: wind magnetic field data in RTN coordinates, displayed similarly to that of Solar Orbiter. Bottom panel: proton speed (black) and density (red) measured by Wind.

The shock front (delineated by the solid vertical line in Fig. 1) driven by the CME was first observed in situ by Solar Orbiter at 05:06 UT on 19 April 2020 whilst the spacecraft was located at a heliocentric distance of $0.809 \mathrm{AU}$. The shock was then observed by Wind (0.996 AU) at 01:34 UT on 20 April 2020, arriving with a mean proton speed of $346 \mathrm{~km} \mathrm{~s}^{-1}$.

The in situ data reveal a clear magnetic flux rope structure, constrained by the vertical dashed lines in Fig. 1, where the leading edge times of the magnetic flux rope are 08:59 UT on 19 April 2020 at Solar Orbiter and 07:56 UT on 20 April 2020 at Wind (defined by Davies et al. 2021). The CME can be classified as a magnetic cloud, meeting criteria detailed by Burlaga et al. (1981). The configuration of the magnetic flux rope can be classified as south-east-north (Bothmer \& Schwenn 1998; Mulligan et al. 1998) and, therefore, has left-handed chirality and the flux rope axis is of low inclination to the ecliptic plane.

The maximum magnetic field strength reached within the magnetic flux rope was $20.1 \mathrm{nT}$ at Solar Orbiter and $16.3 \mathrm{nT}$ at Wind. This is a relatively high field strength for an ICME at solar minimum and/or from quiet Sun regions. The proton density of the magnetic flux rope measured at Wind was relatively low throughout, with a mean density of $11.6 \mathrm{~cm}^{-3}$; however, this includes the rise in density towards the trailing edge (discounting this rise, the mean density in the flux rope is $8.3 \mathrm{~cm}^{-3}$ ). The rise in density towards the trailing edge is likely due to a compression of the magnetic flux rope caused by the high speed stream that follows, which we observe at Wind to have a mean proton speed 

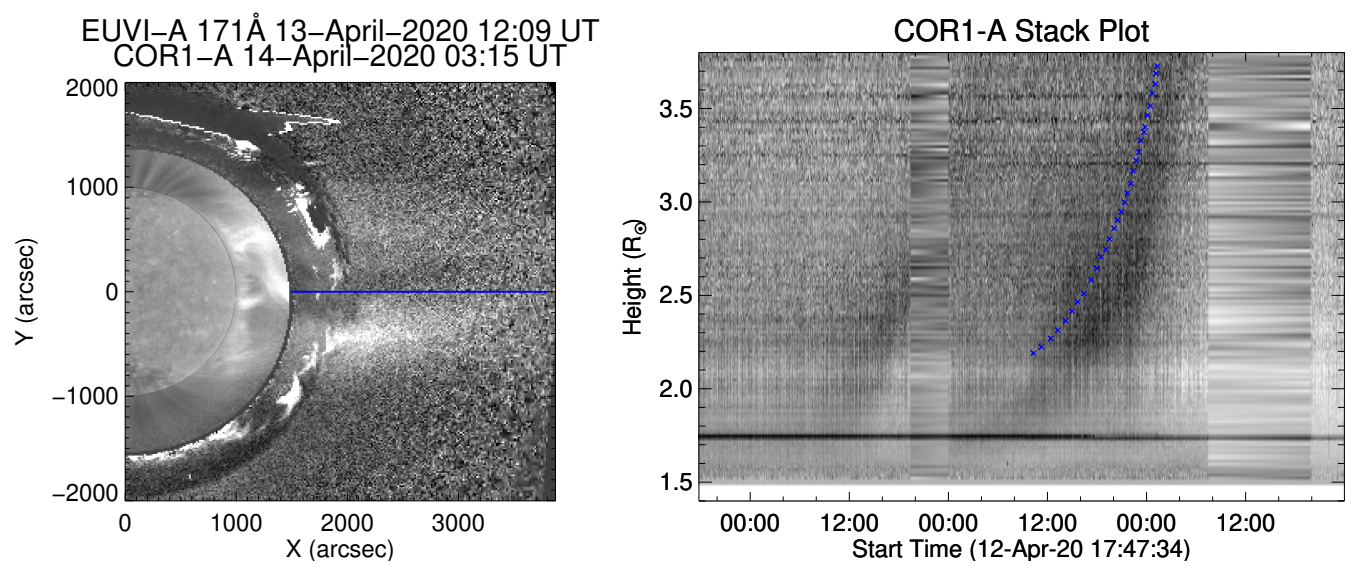

Fig. 2. Left: combined EUVI-A $171 \AA$ and COR1-A, illustrating the connectivity between the high altitude structures observed in EUVI-A and the CME observed in COR1-A. The EUVI-A image was taken at 22:09 UT 13 April 2020, when the cavity was observed, whilst the COR1-A image was taken at 03:15 UT 14 April 2020, when the CME eruption was underway. The blue line represents the $90^{\circ}$ slice taken for the stack plot. Right: stack plot inverse image of COR1-A, taken at an angle of $90^{\circ}$, showing the CME curve. The blue crosses indicate the points selected to track the CME curve.
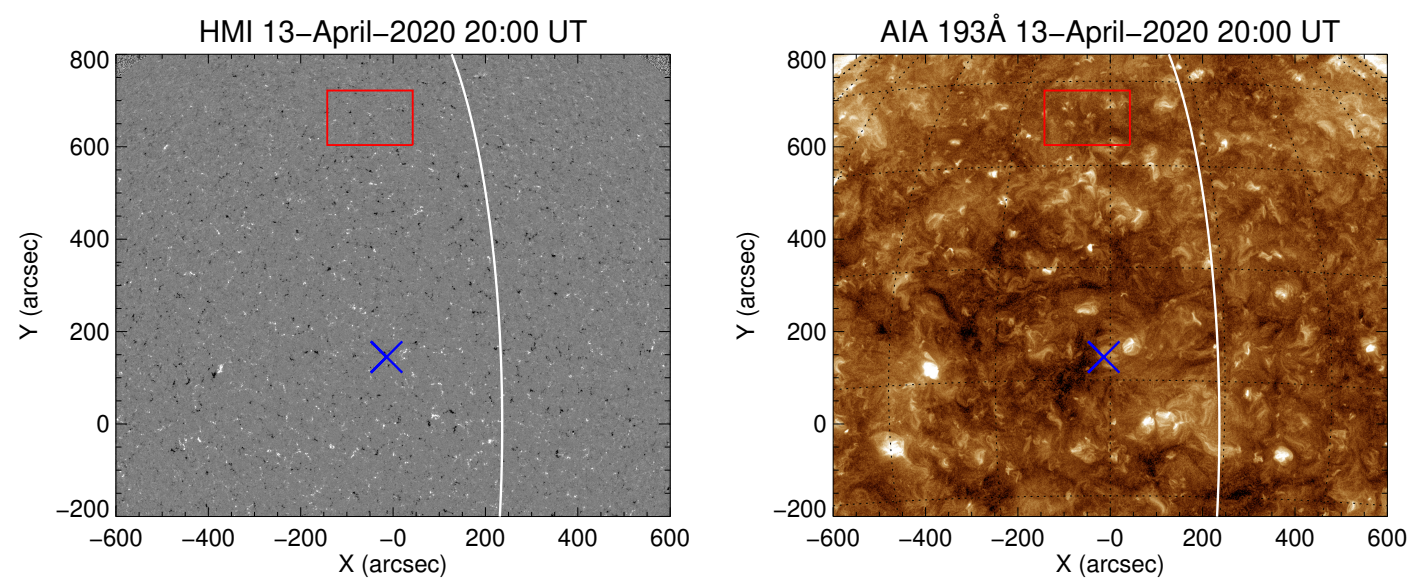

Fig. 3. Left: HMI image at 20:00 UT 13 April 2020, showing the magnetic field just before the cavity began to rise. The blue ' $\mathrm{X}$ ' marks the location of the GCS approximated source region, whilst the red box marks the approximate location of the cavity that was observed at the solar limb. Right: corresponding AIA $193 \AA$ Amage, with the GCS and cavity regions being highlighted as before. In both figures, the limb of EUVI-A is over-plotted as the white line.

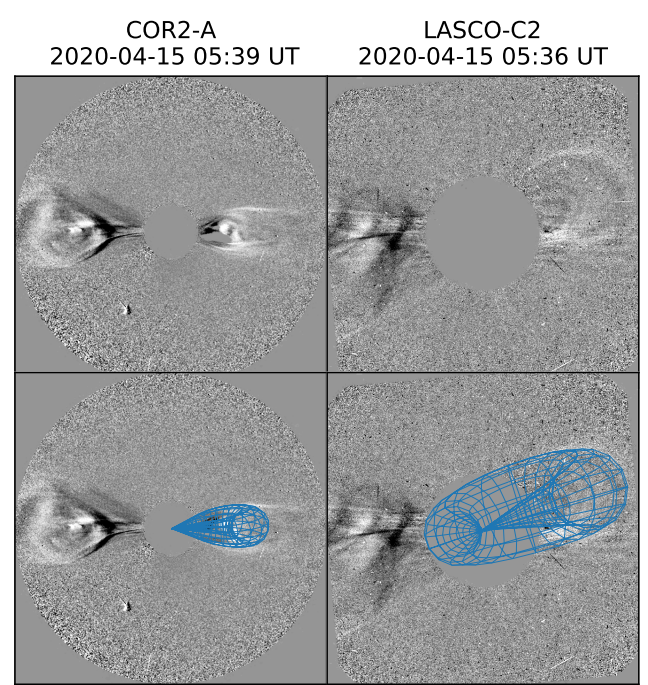

Fig. 4. Remote sensing observations of the CME. STEREO-A COR2 (direct images) and SOHO LASCO C2 (running difference images). Bottom panels: the GCS fitting according to the parameters obtained by Freiherr von Forstner et al. (2021) is indicated by the blue mesh. of $\sim 450 \mathrm{~km} \mathrm{~s}^{-1}$. The high speed stream may also be the reason we observe a stronger magnetic field strength than expected within the CME, where the mean magnetic field strength of the flux rope (18.4 nT at Solar Orbiter and 13.6 nT at Wind) is much stronger than those calculated for ICMEs at similar heliocentric distances (e.g., Janvier et al. 2019).

The magnetic field profiles observed at both spacecraft show a slight decline in magnetic field strength as the flux rope passes over the spacecraft. Such asymmetric profiles are consistent with the model of an expanding flux rope (Nieves-Chinchilla et al. 2018; Janvier et al. 2019). However, the proton speed profile of the CME observed at Wind shows only a small decline throughout the magnetic flux rope. Asymmetric magnetic field profiles with a relatively small expansion may correspond to ICMEs with distorted structures (Nieves-Chinchilla et al. 2018), as observed in images taken by the STEREO-A Heliospheric Imager (HI) of this event (Davies et al. 2021). The mean propagation speed of the leading edge between Solar Orbiter and Wind was $341 \mathrm{~km} \mathrm{~s}^{-1}$, assuming a constant transit velocity would suggest a CME onset time $\sim 5$ days prior to its arrival at Wind (i.e., on 15 April 2020).

We have applied a semi-empirical 3D flux rope model to the Solar Orbiter data (3DCORE, Möstl et al. 2018; 

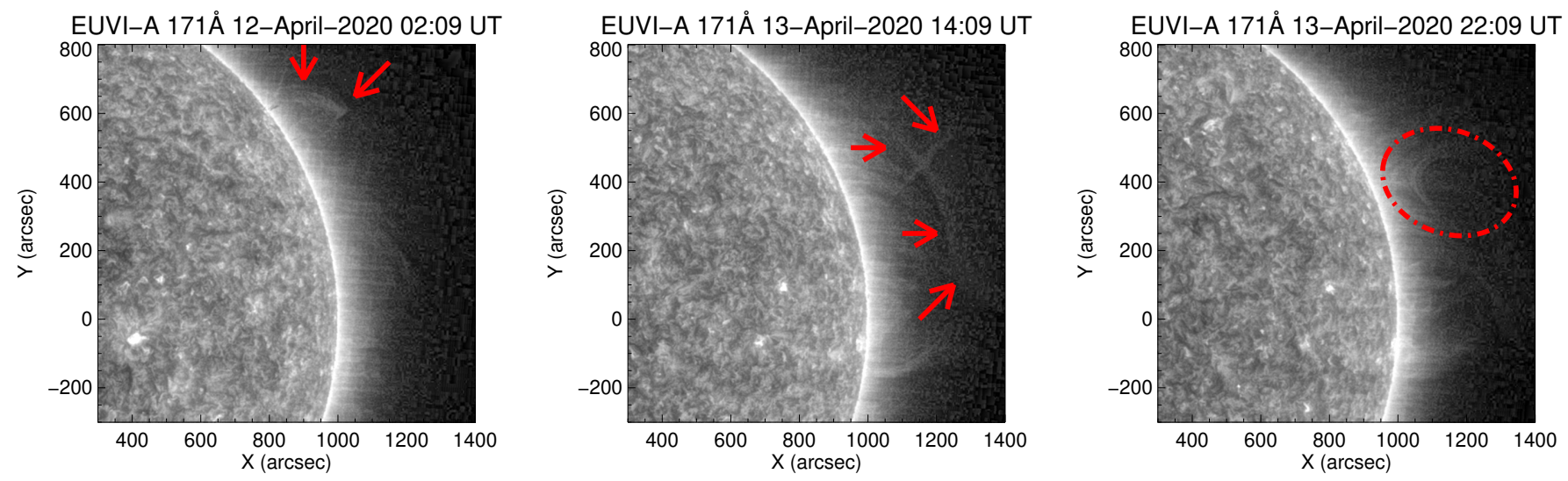

Fig. 5. MGN processed EUVI-A $171 \AA$ Amages, illustrating the structures contributing to the stealth CME. Left panel: the red arrows point out a bright loop structure that is above filament material. Middle panel: large bright high altitude structure, illustrated by the red arrows. Right panel: cavity structure, highlighted by the red-dashed circle.

Weiss et al. 2021a) in order to derive global parameters of the flux rope within this ICME. Detailed results are given in Davies et al. (2021) and Freiherr von Forstner et al. (2021), and work on a paper on simultaneous multi-point fitting of the Solar Orbiter, Wind, and BepiColombo flux rope magnetic field data is underway (Weiss et al. 2021b). Here we present additional information on the magnetic flux derived from the 3DCORE fit on the Solar Orbiter MAG data only. This is of particular interest because for stealth CMEs, large-scale magnetic reconnection during flares can be ruled out as a formation mechanism of the flux rope (e.g., Qiu et al. 2007; Möstl et al. 2009), and this yields parameters of a flux rope that was pre-existing in the solar corona.

Davies et al. (2021) report the fit results with the 3DCORE technique applied to the Solar Orbiter data, propagated to $1 \mathrm{AU}$ : an axial field strength of $B_{0}=14.3 \pm 0.9 \mathrm{nT}$, a flux rope diameter of $D_{1 \mathrm{AU}}=0.114 \pm 0.022 \mathrm{AU}$, and a twist of $\tau=-3.7 \pm 0.6$ field line turns over the full torus $(\tau<0$ implies left-handed chirality), or $\tau_{1 \mathrm{AU}}=-0.6 \pm 0.1$ turns per AU. We have numerically integrated the flux rope cross section to find an axial flux of $\Phi_{\mathrm{ax}}=0.30 \pm 0.06 \times 10^{21} \mathrm{Mx}$. The application of the formula for a uniform twist cylindrical flux rope (e.g., Eq. (16) in Vandas \& Romashets 2017), with the parameters derived from the 3DCORE fit,

$\Phi_{\mathrm{ax}}=\frac{\pi B_{1 \mathrm{AU}}}{\tau_{1 \mathrm{AU}}} \ln \left(1+\tau_{1 \mathrm{AU}} D_{1 \mathrm{AU}} / 2\right)$,

gives $\Phi_{\mathrm{ax}}=0.33_{-0.13}^{+0.16} \times 10^{21} \mathrm{Mx}$, which is consistent with the numerical result; although, this should in general be seen only as a rough approximation because the flux rope cross section in 3DCORE is not cylindrical, but elliptical. The axial flux we determined for this event is thus at the lower end of reported axial fluxes in ICME flux ropes (cf. Qiu et al. 2007).

\subsection{Stealth $C M E$ source region}

As seen in Fig. 2, the stealth CME is observed in COR1-A as a faint enhancement in density, and it becomes clear in COR2A by 03:54 UT 15 April 2020, following the brightening and swelling of a streamer. The CME exhibits a bright underside and a clear concave-up structure, which is suggestive of a flux rope configuration (Vourlidas et al. 2013) . In COR1-A, the underside of the CME can be clearly tracked from 10:16 UT 14 April 2020 (Fig. 2), using the $90^{\circ}$ slice, and it has an average velocity

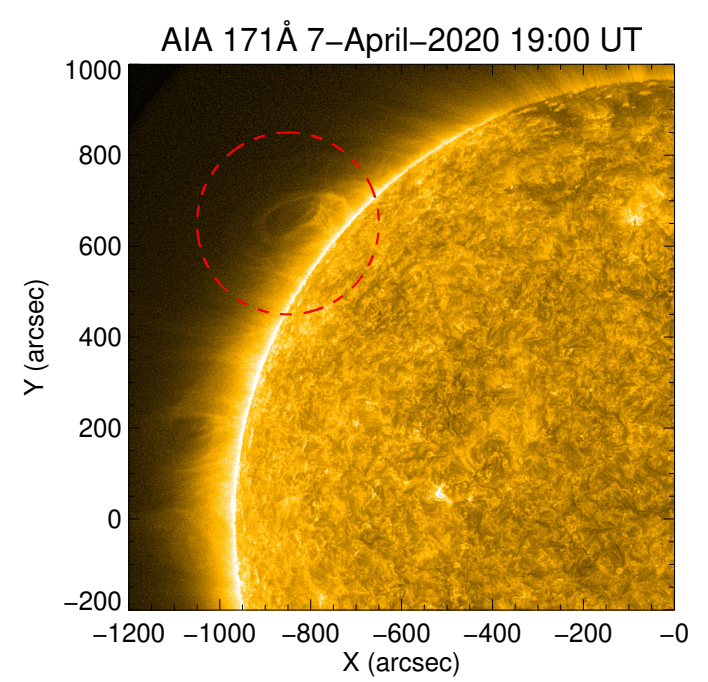

Fig. 6. MGN-processed AIA $171 \AA$ image at 19:00 UT 7 April 2020, showing the cavity structure observed on the limb (red-dashed circle) $\sim 6$ days before the eruption.

of $18 \mathrm{~km} \mathrm{~s}^{-1}$. Using this speed and a height of $2.5 R_{\odot}$ at $22: 30 \mathrm{UT}$ 14 April 2020, we estimate a more accurate CME onset time of 19:30 UT 13 April 2020. In COR2-A, the CME has an average speed of $53 \mathrm{~km} \mathrm{~s}^{-1}$. The increase in CME speed between that measured from COR2-A and that measured in situ at Wind is likely due to the high speed stream following the CME and the background solar wind.

Primary observations of the Sun during this time period show no active regions, and the photospheric magnetic field exhibited only weak small-scale field fragments (see Fig. 3). EUV and $\mathrm{H} \alpha$ data showed that no filaments or filament channels were present either, making the source region of the $\mathrm{CME}$ very challenging to identify. Using the images from COR2-A and C2 at 05:39 UT and 05:36 UT 15 April 2020, respectively, the CME was fitted with the GCS model (see Fig. 4). The GCS fit found an approximate source region at $143 \pm 7^{\circ}$ longitude and $3 \pm 3^{\circ}$ latitude (see Table 2 in Freiherr von Forstner et al. 2021), and it is indicated by the blue cross in Fig. 3. This puts the approximate source region on disk from SDO, and on limb from STEREO-A.

Although both the MGN and difference imaging techniques were applied to each AIA passband, they did not reveal any clear eruption signatures on disk. However, the NRGF and MGN 

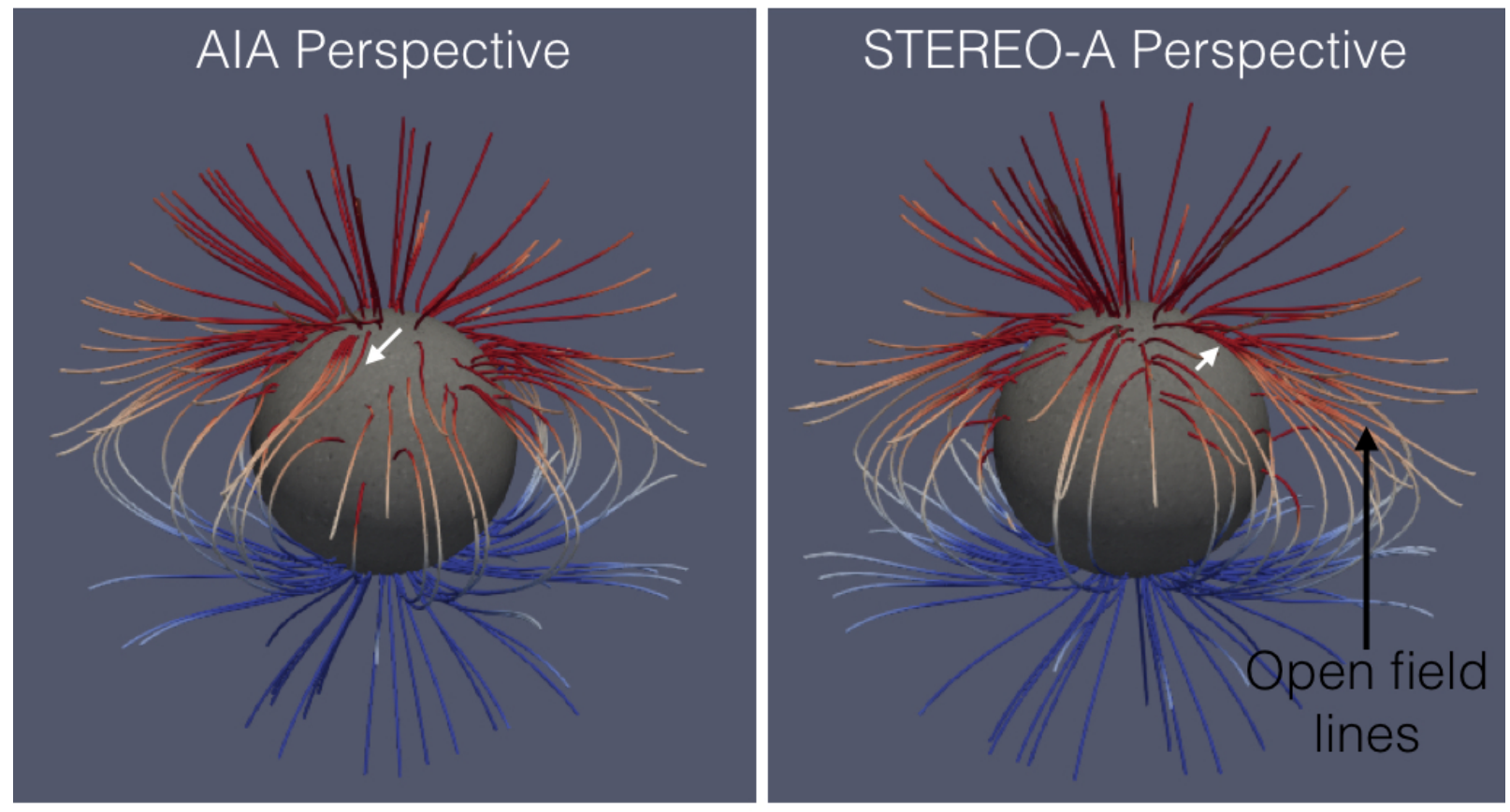

Fig. 7. PFSS model of Carrington rotation 2229, as observed from the AIA perspective (left) and STEREO-A perspective (right). Positive (negative) polarity is coloured in red (blue), on 13 April 2020. The approximate source region of the stealth CME is indicated by the white arrows.

processed $195 \AA$ and $171 \AA ̊$ EUVI-A data revealed multiple highaltitude dynamic structures off the solar limb, including a bright looped region, a large bright structure, and a cavity structure (Fig. 5). The large bright structure is observed from 08:50 UT 13 April 2020, and then it slowly rises into COR1-A, coinciding with the brightening and swelling of the streamer. The concaveup cavity structure is observed from 22:09 UT 13 April 2020 at $35.7^{\circ}$ latitude, and it is deflected southwards as it erupts from the Sun, coinciding with initiation of the CME in COR1-A. We therefore deduce that this is the cavity responsible for the CME that is later detected in situ. The deflection of the cavity was measured by applying the GCS model to the structure in $171 \AA$ EUVI-A. The cavity is observed to deflect southwards by $\sim 19^{\circ}$ within the EUVI FOV, and a further $\sim 12^{\circ}$ into the FOV of COR1. It is noted that due to applying the GCS model to one spacecraft instead of two, errors are larger. However, this is smaller for latitude such that the deflection can be measured with reasonable confidence.

The cavity is an observational signature of a flux rope present in the low corona as the eruption takes place. We tracked the region back to the eastern limb of SDO to look for evidence of the flux rope present prior to the eruption. Three cavities are observed on the limb in AIA 171 A between 6 and 10 April 2020. The first cavity at $10^{\circ}$ latitude erupted off limb on 8 April 2020, and therefore was ruled out as being the source. The second cavity at $40^{\circ}$ latitude, which is highlighted by the red-dashed circle in Fig. 6, closely matches the erupting cavity observed in EUVI-A (at $35.7^{\circ}$ latitude) and with its approximate longitude. The third cavity at $30^{\circ}$ latitude does not match in longitudinal extent with the cavity observed at the limb of EUIV-A. Thus we conclude the second cavity observed is the structure that later erupts as the stealth CME. Using these two observations, we obtain an approximate source region from the SDO perspective as $139^{\circ} \pm 5^{\circ}$ longitude and $38^{\circ} \pm 2^{\circ}$ latitude, indicated by the red box in Fig. 3. The difference between the source region and the estimated region obtained from the GCS is due to the southwards deflection of the cavity as it erupts.
We further investigated the magnetic topology of the source region and its surrounding environment with a potential field source surface (PFSS) model, using the PFSS solver in Python (pfsspy, Yeates 2018; Stansby 2019). Figure 7 shows the PFSS model as viewed from both the AIA (left) and STEREO-A (right) perspectives, with the modelled magnetic field lines showing the positive (red) and negative (blue) polarities. The CME originates in the northern hemisphere, near the central meridian (west limb) from the AIA (STEREO-A) perspective. Open field lines are deflected southwards, towards the equator, similar to the trajectory of the cavity observed in EUVI-A. Given the absence of active regions and the solar minimum phase at this time, the global magnetic field is close to that of a dipole field.

\section{Discussion and conclusions}

Here, we present a study of the first stealth CME observed in situ by Solar Orbiter, with accompanying Wind in situ observations and STEREO-A and SDO remote sensing data. The combined use of STEREO-A and SDO data revealed that the origin of the CME was a quiet Sun cavity within small-scale magnetic field and that there was no clear polarity inversion line. The cavity identified in EUVI-A and AIA $171 \AA$ A passbands extended up to $1.3 R_{\odot}$ (from Sun centre) in the corona. Such dark elliptical cavities in the quiet Sun indicate the presence of a magnetic flux rope and have been found to have a median height of $1.2 R_{\odot}$ (Gibson 2015).

Despite the quiet Sun origin of the stealth CME, the magnetic cloud still had a very high field strength in the interplanetary medium (13.6 $\mathrm{nT}$ at Wind). The $\sim 1 \mathrm{AU}$ value is significantly higher than that found in a comparison study of stealth CMEs at solar minimum by Kilpua et al. (2014), who show an average $B_{\max }$ of $9.7 \mathrm{nT}$ across a sample of ten stealth CMEs, and the in situ stealth CME study of Nieves-Chinchilla et al. (2013). This maximum field strength and the south-east-north configuration of the flux rope may have been the deciding 
factors in this event producing a category $\mathrm{G} 1$ geomagnetic storm. The slow speed of stealth CMEs means that we do not typically expect them to drive shocks. In this event, a forward shock is detected in situ, similar to the case of the stealth CME studied in Nieves-Chinchilla et al. (2013). The shock is likely due to the slower solar wind conditions at $1 \mathrm{AU}$ ahead of the ICME $\left(\sim 300 \mathrm{~km} \mathrm{~s}^{-1}\right)$. Also, the slight expansion in the unperturbed section of the ICME means that a combination of a relatively slow solar wind speed ahead of the CME and the CME expansion may drive the shock as proposed by Lugaz et al. (2017).

This study reinforces the need for multi-wavelength, multiview point observations of the solar corona, combined with image processing techniques, to further our ability to detect and analyse stealth CME source regions. These observations indicate that for Solar Orbiter EUI to successfully observe stealth $\mathrm{CME}$ sources, observing sequences should include regular synoptic images from the $174 \AA$ waveband of the Full Sun Imager (FSI) telescope, which will have a field-of-view of $4 R_{\odot}$ at perihelion.

Acknowledgements. JO thanks the STFC for support via funding given in her PHD studentship. LMG is grateful to the Royal Society, which supported this research through the Royal Society University Research Fellowship scheme. DML is grateful to the Science Technology and Facilities Council for the award of an Ernest Rutherford Fellowship (ST/R003246/1). JvF thanks the German Space Agency (Deutsches Zentrum für Luft- und Raumfahrt e.V., DLR) for their support of his work on the Solar Orbiter EPD team under grant 50OT2002. CM, AJW, JH and TA thank the Austrian Science Fund (FWF): P31521-N27, P31659N27, P31265-N27. ED is supported by funding from the Science and Technology Facilities Council (STFC) studentship ST/N504336/1. SDO is a mission of NASA's Living With a Star Program. STEREO is the third mission in NASA's Solar Terrestrial Probes program. SOHO is a mission of international cooperation between ESA and NASA. The authors thank the SDO, STEREO, and SOHO teams for making their data publicly accessible. The Solar Orbiter magnetomete was funded by the UK Space Agency (grant ST/T001062/1). Its data are available in the Solar Orbiter Archive at http://soar. esac. esa.int/soar/.

\section{References}

Alzate, N., \& Morgan, H. 2017, ApJ, 840, 103

Bothmer, V., \& Schwenn, R. 1998, Ann Geophys., 16, 1

Brueckner, G., Howard, R., Koomen, M., et al. 1995, in The Large Angle Spectroscopic Coronagraph (LASCO), The SOHO Mission (New York: Springer), 357

Burlaga, L., Sittler, E., Mariani, F., \& Schwenn, R. 1981, J. Geophys. Res. Space Phys., 86, 6673

Byrne, J., Long, D., Gallagher, P., et al. 2013, A\&A, 557, A96
Davies, E. E., Möstl, C., Owens, M. J., et al. 2021, A\&A, 656, A2 (SO Cruise Phase SI)

D’Huys, E., Seaton, D., Poedts, S., \& Berghmans, D. 2014, ApJ, 795, 49

Domingo, V., Fleck, B., \& Poland, A. I. 1995, Sol. Phys., 162, 1

Freiherr von Forstner, J. L. 2021, https://doi.org/10.5281/zenodo. 4443203

Freiherr von Forstner, J. L., Dumbović, M., Möstl, C., et al. 2021, A\&A, 656, A1 (SO Cruise Phase SI)

Gibson, S. 2015, in Coronal Cavities: Observations and Implications for the Magnetic Environment of Prominences, eds. J. C. Vial, \& O. Engvold (Cham: Springer), Astrophys. Space Sci. Lib., 415, 323

Green, L., Torok, T., Vršnak, B., et al. 2018, Space Sci. Rev., 214, 46

Horbury, T. S., O’Brien, H., Carrasco Blazquez, I., et al. 2020, A\&A, 642, A9

Howard, T. A., \& Harrison, R. A. 2013, Sol. Phys., 285, 269

Howard, R. A., Moses, J. D., Vourlidas, A., et al. 2008, Space Sci. Rev., 136, 67

Janvier, M., Winslow, R. M., Good, S., et al. 2019, J. Geophys. Res. Space Phys., 124,812

Kaiser, M. L., Kucera, T., Davila, J., et al. 2008, Space Sci. Rev., 136, 5

Kilpua, E., Mierla, M., Zhukov, A., et al. 2014, Sol. Phys., 289, 3773

Lemen, J. R., Akin, D. J., Boerner, P. F., et al. 2012, Sol. Phys., 275, 17

Lepping, R. P., Acũna, M. H., Burlaga, L. F., et al. 1995, Space Sci. Rev., 71, 207

Lugaz, N., Farrugia, C. J., Winslow, R. M., et al. 2017, ApJ, 848, 75

Morgan, H., \& Druckmüller, M. 2014, Sol. Phys., 289, 2945

Morgan, H., Habbal, S. R., \& Woo, R. 2006, Sol. Phys., 236, 263

Möstl, C., Farrugia, C. J., Miklenic, C., et al. 2009, J. Geophys. Res. Space Phys., 114, A04102

Möstl, C., Amerstorfer, T., Palmerio, E., et al. 2018, Space Weather, 16, 216

Müller, D., Cyr, O. S., Zouganelis, I., et al. 2020, A\&A, 642, A1

Mulligan, T., Russell, C. T., \& Luhmann, J. G. 1998, Geophys. Rev. Lett., 25, 2959

Nieves-Chinchilla, T., Vourlidas, A., \& Stenborg, G. 2013, ApJ, 779, 55

Nieves-Chinchilla, T., Vourlidas, A., Raymond, J. C., et al. 2018, Sol. Phys., 293, 25

Nitta, N. V., \& Mulligan, T. 2017, Sol. Phys., 292, 125

Ogilvie, K. W., Chornay, D. J., Fritzenreiter, R. J., et al. 1995, Space Sci. Rev., 71,55

O'Kane, J., Green, L., Long, D. M., \& Reid, H. 2019, ApJ, 882, 85

O'Kane, J., Cormack, C. M., Mandrini, C. H., et al. 2021, ApJ, 908, 89

Palmerio, E., Kilpua, E. K., James, A. W., et al. 2017, Sol. Phys., 292, 39

Palmerio, E., Kilpua, E. K. J., Möstl, C., et al. 2018, Space Weather, 16, 442

Patsourakos, S., Vourlidas, A., Török, T., et al. 2020, Space Sci. Rev., 216, 131

Pesnell, W. D., Thompson, B. J., \& Chamberlin, P. 2012, Sol. Phys., 275, 3

Qiu, J., Hu, Q., Howard, T. A., \& Yurchyshyn, V. B. 2007, ApJ, 659, 758

Robbrecht, E., Patsourakos, S., \& Vourlidas, A. 2009, ApJ, 701, 283

Scherrer, P. H., Schou, J., Bush, R., et al. 2012, Sol. Phys., 275, 207

Stansby, D. 2019, https: //doi .org/10.5281/zenodo. 2566462

Thernisien, A., Howard, R., \& Vourlidas, A. 2006, ApJ, 652, 763

Vandas, M., \& Romashets, E. 2017, A\&A, 608, A118

Vourlidas, A., Lynch, B. J., Howard, R. A., \& Li, Y. 2013, Sol. Phys., 284, 179

Weiss, A. J., Möstl, C., Amerstorfer, T., et al. 2021a, ApJS, 252, 9

Weiss, A. J., Möstl, C., Davies, E. E, et al. 2021b, A\&A, 656, A13 (SO Cruise Phase SI)

Yeates, A. 2018, https://doi.org/10.5281/zenodo. 1472183 